\title{
The Group Visit Model-A Promising Option for Clinical Care Delivery in Patients with Chronic Migraine
}

\author{
Laura Wolf and Larisa Syrow \\ Cooper Medical School of Rowan University, Camden, NJ, USA
}

DOl: https://doi.org/10.17925/USN.2020.16.1.19

$(2$ hronic headache is a highly prevalent medical problem that poses challenges to the patient and the healthcare provider. Patients experience debilitating pain, often accompanied by nausea, vomiting, photophobia, and other symptoms on a regular basis. The majority of patients lack access to specialized care and receive suboptimal treatment, especially in areas of low socioeconomic status. Due to the national shortage of neurologists, patients have difficulty locating providers and wait long periods of time for appointments. The medical group visit model is an innovative method for delivery of high-quality clinical care to patients with chronic conditions like headache. We developed a medical group visit model for patients with chronic headache at the Headache Program of the Cooper University Urban Health Institute in Camden, NJ, USA, a medically underserved city with a complex patient population. Key elements of the model include brief patient-doctor meetings, group discussions facilitated by medical staff or specialty-trained guest speakers, stress management education (such as meditation and yoga), as well as administration of nerve blocks and botulinum toxin (Botox) injections. Implementation of the model has produced personalized patient-doctor encounters, supportive peer-peer interaction, patient education, decreased appointment lag time, increased physician productivity and cost-effectiveness.

\section{Keywords}

Medical group visit, shared appointment, chronic headache, headache management, migraine, chronic pain, counseling, cost-effectiveness

\section{Disclosure: Laura Wolf and Larisa Syrow have no financial or non-financial relationships or activities to declare in relation to this article. Review Process: Double-blind peer review. Compliance with Ethics: This special report describes clinical experience utilizing a group visit model. It is not research per se, so did not require ethics approval. The name of the patient who provided feedback on the headache group has been changed to protect their privacy. Authorship: The named authors meet the International Committee of Medical Journal Editors (ICMJE) criteria for authorship of this manuscript, take responsibility for the integrity of the work as a whole, and have given final approval for the version to be published. \\ Access: This article is freely accessible at touchNEUROLOGY.com () Touch Medical Media 2020. \\ Received: May 3, 2019 \\ Accepted: November 4, 2019 \\ Citation: US Neurology. 2020;16(1):19-24 \\ Corresponding Author: Laura Wolf, Cooper Medical School of Rowan University, 401 South Broadway, Camden, NJ 08103, USA. E: wolflo@rowan.edu}

Support: No funding was received in the publication of this article.
The medical group visit model is an innovative mode of care delivery that has gained popularity with clinicians, as it offers more options for chronic care management, with the added possibility of improving financial productivity. Group medical visits were initially developed in the 1970s in an effort to offer high-quality medical care with additional elements of patient support. Typically, a visit involves multiple patients assembling together with providers for an expanded, augmented version of the individual appointment. Key elements of the model include brief patient-doctor meetings, group discussions facilitated by medical staff or specialty-trained guest speakers, stress management education (such as meditation and yoga), as well as administration of nerve blocks and botulinum toxin (Botox) injections.

In the era of a shortage of adult headache specialists/neurologists, the headache group medical visit provides a viable model for increased access to care, comprehensive patient education and counseling, and increased provider efficiency. Here we describe our implementation of group visit model care for patients suffering with chronic headache within a resource-poor community in Camden, NJ, USA. The model described below continues to evolve in its format to meet productivity expectations and improve quality service care.

Maria DelSanto (name changed to protect the privacy of the individual), a 50-year-old female patient from Camden, NJ, USA, who struggles with chronic migraine with vertigo, is a regular attendee of the Cooper University Urban Health Institute headache group. She enjoys the social aspect of the visits, stating "the group helps me to relate to other people who have migraines and a lot of pain, like I do. I like to hear other people talk about it, because then I don't feel alone." Maria actively participates in group discussion and shares her opinion on the different headache medical therapies, such as Botox, nerve blocks, and the new self-injectable calcitonin gene-related peptide (CGRP) antagonist, Aimovig® (Amgen, Thousand Oaks, CA, USA). She speaks about the utility of stress-management techniques to relieve headache pain: "after we did the meditation class here, I went home and used one of those meditation videos to help me fall asleep that night. I felt so relaxed!"

Treatment of primary headache disorders presents a challenge to healthcare providers. The estimated prevalence of headache disorders in adults (symptomatic at least once per year) is approximately $50 \%$ worldwide. Of those, $30 \%$ have reported migraine. ${ }^{1}$ Headache disorders are 
classified as either primary or secondary. Primary headache syndromes include migraine, tension-type headache, and trigeminal autonomic cephalalgias, and other less common conditions such as primary cough headache, exertional headache, and primary stabbing headache. Tension-type headache and migraine are the second and third most prevalent disorders in the world (after dental caries); migraine is the seventh highest cause of disability in the world. ${ }^{2}$ Migraine typically affects people between 15-49 years of age and is more common in women. There are several subtypes of migraine, including menstrual migraine, hemiplegic migraine, vestibular migraine, and chronic migraine. To receive a diagnosis of chronic migraine, a patient experiences a headache occurring at least 15 days per month for at least 3 months. The headache will have migraine features such as aura, unilaterality, pulsatile quality, nausea and vomiting, photophobia, phonophobia, cutaneous allodynia, and duration of hours to days. ${ }^{3}$ Secondary headache disorders are due to medication overuse, cervical spine disease, expansive intracranial processes, and underlying systemic conditions such as fever, hypertension, pregnancy, or sinusitis.

Headaches diminish quality of life, decrease job and social functioning, and increase utilization of healthcare resources. ${ }^{4}$ The majority of patients with headaches do not have access to specialized care. A recent study identified only 416 headache specialists certified by the United Council for Neurological Subspecialties currently practicing in the USA; whereas, there are about 37 million Americans over age 12 suffering with migraine. ${ }^{5}$ This is a drastic disparity between headache subspecialists and the extensive migraine population. Lack of access to optimal treatment may lead to unnecessary visits to emergency departments and urgent care facilities, which increases the overall cost of care. ${ }^{6}$

An association has been established between low socioeconomic status and higher risk for migraine.? Furthermore, an association has been suggested between low socioeconomic status and chronification of headaches. ${ }^{8.9}$ To address the challenges facing care for patients with chronic headache, from a low socioeconomic background, we established a headache group visit model at the Cooper University Urban Health Institute in Camden, NJ. Camden City has a medically underserved, resource-poor population that is largely African American and Hispanic.10 Our patient population with headaches is medically complex, with high rates of comorbidity with depression, anxiety, and other chronic pain disorders (such as fibromyalgia, neck/back pain). The Urban Health Institute was established to provide innovative care in this resource-constrained practice.

\section{Structure}

The headache group visit clinical team consists of a board-certified neurologist specializing in headache medicine, a physician assistant with extensive experience in headache management, licensed practical nurses, and medical assistants. The advanced practice nurse, licensed practical nurses, physician assistant, and clinical pharmacist serve as patient navigators. Occasionally, other healthcare providers, such as rotating neurology residents, serve in the role of navigators. Rotating medical students help facilitate by assisting with vital sign collection, obtaining information from outside sources, participating in group education discussion, and by offering individual counseling.

To join a group visit, patients must be referred to the clinic by a neurologist at Cooper University Hospital. Prior to attending a group session, patients are required to have an individual appointment with the headache neurologist to rule out secondary causes of headache. The majority of our patients are diagnosed with chronic migraine. If appropriate, the patient is offered the opportunity to be seen at group visits for future appointments. Participation in the group is voluntary. The headache group visit is conducted for a 2-hour session, once per month. Up to 18 patients are scheduled with expected show-rate of 7-15 patients. Private exam rooms are available for patients, if needed. The predominant components of the group are medical management; learning lifestyle modification for headache reduction, including yoga and meditation; facilitated group discussion; and injection administration (nerve block and Botox). Figure 1 illustrates the process of a group visit.

\section{Bilingual accommodations}

The visits accommodate patients whose primary language is Spanish. We do this by offering translation via Spanish-speaking licensed practical nurses, medical assistants, and the neurologist. The group discussions are conducted in both English and Spanish.

\section{Recording medical information}

Prior to the group, visit a staff member will put together a "huddle sheet" (Figure 2), which is a form that keeps all the relevant patient information to hand for the group visit. The form gives staff a snapshot of the patient's medical history, current medication, prior imaging, last administered injections, recent changes in management, and information on psychiatric treatments. These forms help expedite the patient interviews and clinical decision making. The information that is updated on the huddle sheet may be used for note writing on the electronic medical record.

Upon arrival to the group visit, patients check in with medical assistant and are escorted into the group education room where vital signs are taken. This room is dimly lit for the sake of those with photophobia. Sometimes light, low-volume meditative music is played in the background. While some patients are waiting to be seen by the clinical team, other patients are presented with meditative imagery and relaxing sounds. During the waiting period, mediators, such as medical students, volunteers, or other personnel individually discuss patient goals for the visit and address questions. While patients are waiting to speak to a member of the medical team, they are also encouraged to interact with each other. For example, if one patient is about to start a new treatment for the first time and another has had it many times in the past, they are encouraged to discuss the procedure and share stories. This is also an opportunity for patients to develop bonds. Some offer carpooling, and some exchange phone numbers for continued mutual support. During waiting times, we occasionally have a social worker come in to offer brief individual counseling to patients in acute distress.

Navigators call patients into the navigation room one-by-one to be interviewed. During the interview the navigators inquire about frequency of headaches, adequacy of treatment, stress, and other medical conditions. When the physician is ready to see the patient, the navigators "present" patient information and document the management decisions the physician makes. Upon completion of individual interviews, everyone takes part in a group discussion (outlined in the next section). After the interactive discussion, the group learns about, and participates in, guided meditation/relaxation techniques, stretching, or chair yoga exercises (using online resources such as various YouTube videos). 

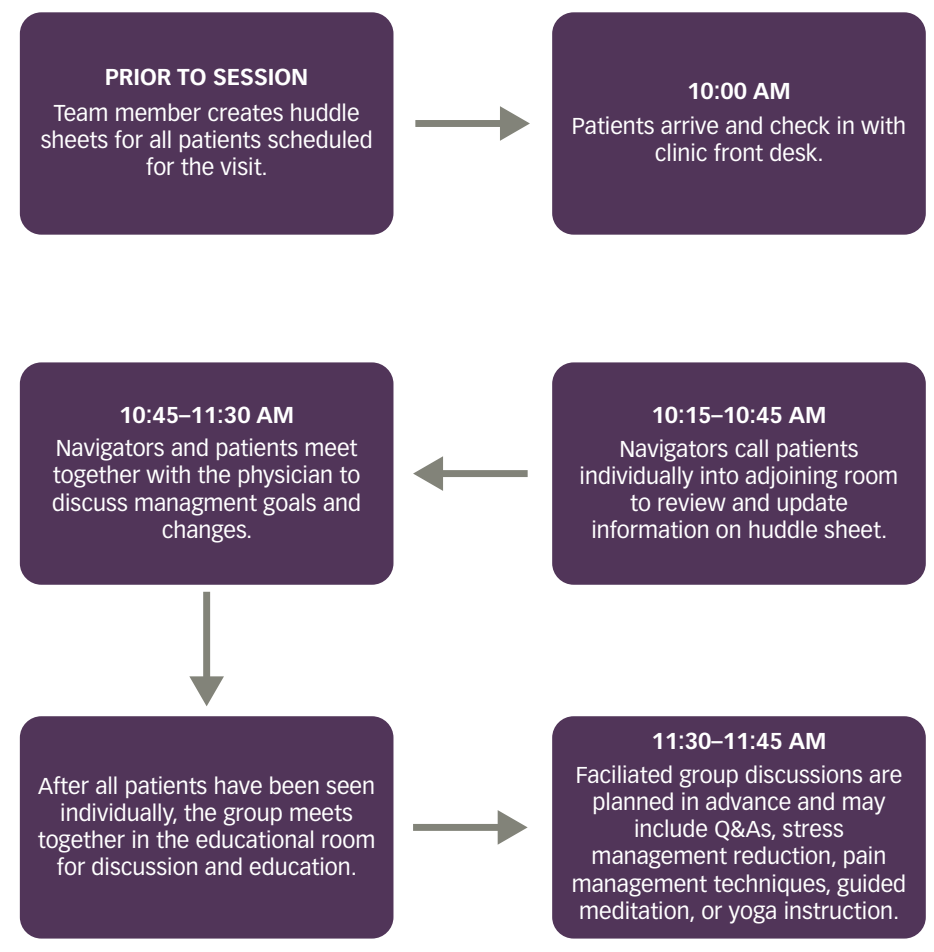

Patients are directed to group

educational room, where they

may sit and interact until called

by patient navigators. Medical

students and residents may

speak with patients during this time.
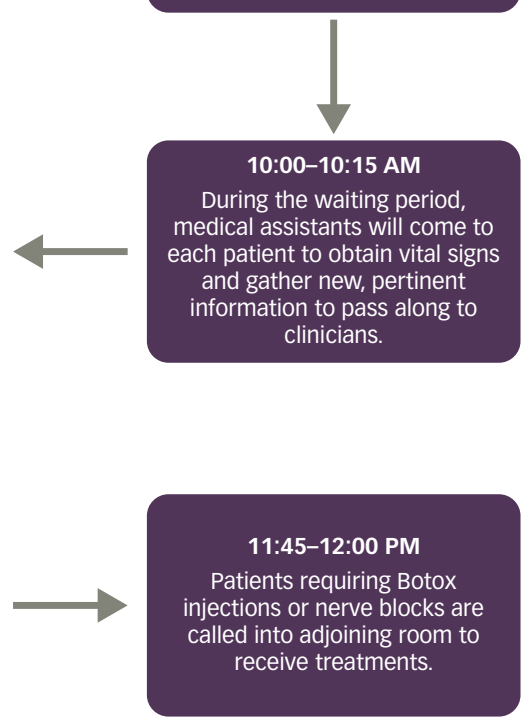

\section{Facilitated group discussion and patient education}

Common discussions include lifestyle modifications such as adequate hydration, practicing good sleep hygiene, keeping a headache diary, and tracking food triggers. The goal is to provide patients with alternative tools to lessen headaches and to encourage them to educate and motivate each other. During these sessions, lists of food triggers and copies of headache diaries are given out. Another goal of the group is to help patients see that they are not alone in suffering with migraines and to facilitate a support-group dynamic where they help each other cope. Many of the patients are able to offer a mutual understanding, given that they have the psychosocial difficulties that are often seen in the Camden population.

Patients also submit questions, written on anonymous index cards, to be addressed by the neurologist in front of the entire group. Common questions are often about the cause of migraine, how to quench a severe migraine and how to avoid medication overuse. Frequent discussion is also about what to do when a severe migraine comes. Patients often share with the group the frustration over chronic headaches and receive support from others who have similar experiences. We also address questions surrounding new therapies, such as the CGRP-antagonist self-injectables, and demonstrate proper self-administration technique using models provided by the manufacturers.

On occasion, we have guest speakers mediate group discussion. Prior guest speakers have been behavioral psychologists, social workers, and patients with successful treatment stories. At times, some of the medical students prepare to lead a group discussion on topics such as sleep hygiene or guided progressive muscle relaxation.

\section{Medical decision making and documentation}

Early in the process, the electronic medical record support and clinical team developed a robust documentation system to allow the non-physician staff to record information in the medical chart. All patient medical plans, including proposed changes to therapy, are discussed with the neurologist. Since all the patients in the group are there for follow-up appointments, only a brief, pertinent neurological examination is conducted. Private exam rooms are available in the group visit suite to use when deemed necessary. Navigators use the electronic medical record (EMR) to document answers to pre-set clinical questions which are embedded into a progress note in the EMR. Navigators also order laboratory tests, medications, and specialist referrals for patients. The neurologist signs off on all medication and laboratory orders and notes written by navigators.

\section{Administering nerve blocks and Botox injections}

occipital nerve blocks are offered for patients with migraines and occipital neuralgia for immediate relief and to help reduce the number of medications. They are offered as needed for abortive relief and on a monthly or bimonthly schedule as a preventive measure. Botox injections are conducted according to US Food and Drug Administration (FDA)-recommended 31-site injection guide for patients with chronic intractable migraines.

While the entire group is engaged in guided meditation or chair yoga, certain patients come out to receive nerve blocks or Botox injections. These are not done in a private setting since we feel that patients benefit from witnessing others getting the treatment. This lessens anxiety about novel treatments and encourages patients to share about their experiences. 
Figure 2: The huddle sheet

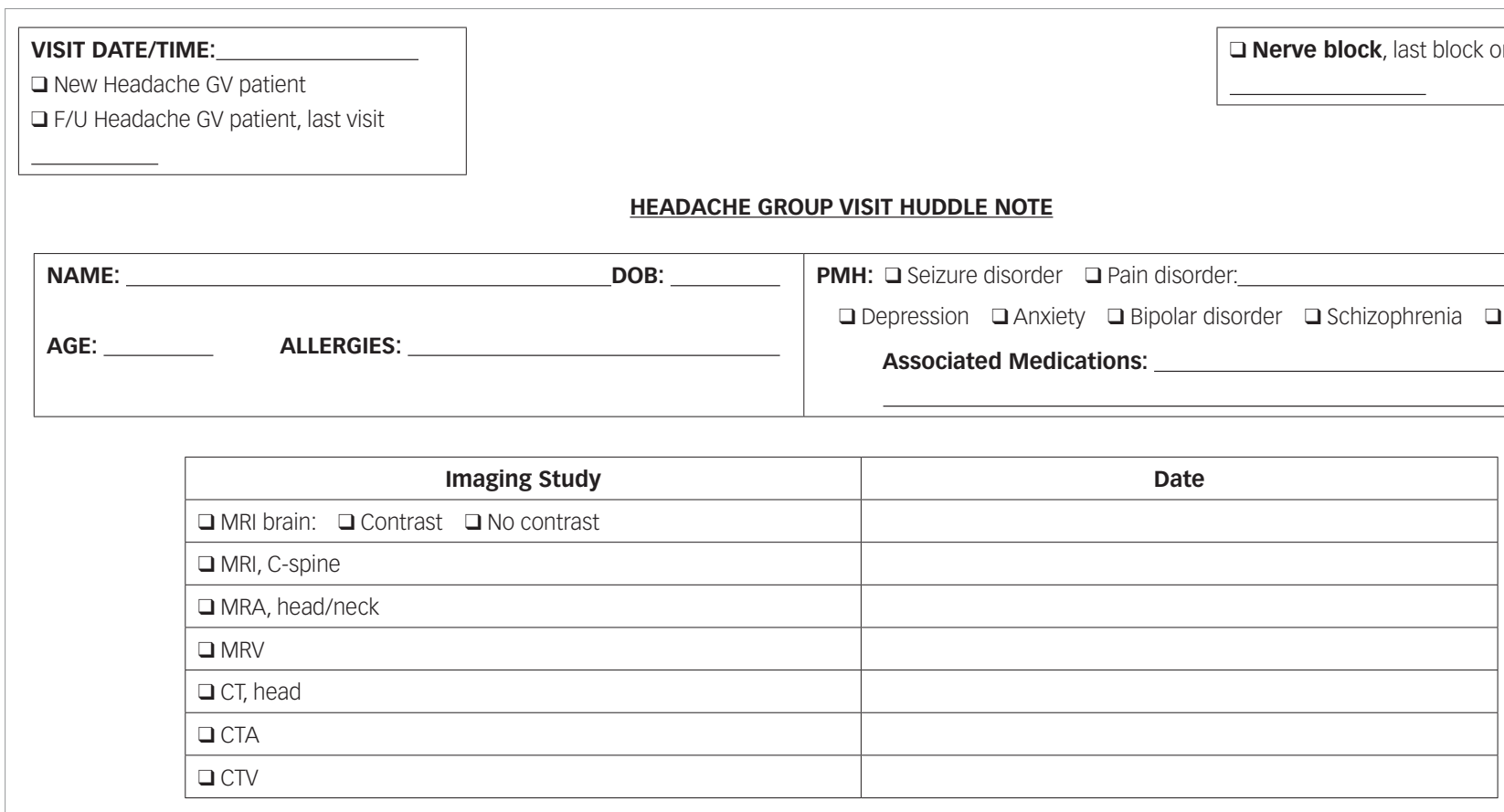

Recent Hospitalization, ED visits, Sugeries, Illnesses:

Current Medications for Headaches:

Changes Made at Last Headache GV:
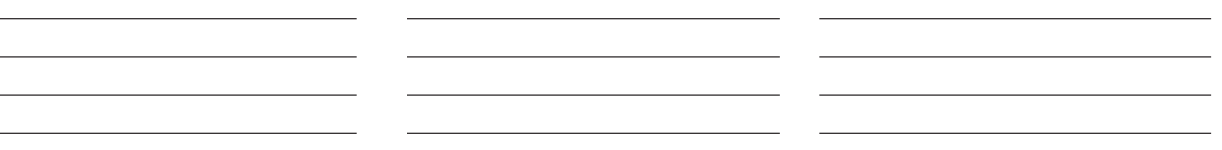

Recent Medication Changes (other than at least headache GV):

Psychosocial Concerns: ] Sees psychiatrist/psychologist, Last visit:

Other Concerns/Recommendations:

The huddle sheet is designed give staff a snapshot of the patient's medical history, current medication, prior imaging, last administered injections, recent changes in management, and information on psychiatric treatments, and is kept on hand during the group visit.

$C T=$ computed tomography; CTA = computed tomography angiography; CTV = computed tomography venography; DOB = date of birth; ED = emergency department; F/U = follow-up;

GV = group visit; $M R A=$ magnetic resonance angiography; MRI = magnetic resonance imaging; $M R V=$ magnetic resonance venography; PMH = past medical history;

PTSD = post-traumatic stress disorder; SX = symptoms. 
The nerve blocks are administered by the neurologist and physician assistant. Botox is administered by the neurologist.

\section{Patient scheduling}

The usual waiting time for an initial neurology appointment is approximately 4 months. The usual period between individual follow-up is also 4 months. Our internal data showed a significant decrease in the expected show rate when patients waited more than 4 weeks for their appointment. With an extended lag time until the next appointment, physicians schedule appointments for patients who will never show. The Urban Health Institute was running financial losses driven by low Medicaid reimbursement rates; a $30-40 \%$ no show rate; and a dependence on a high-cost, poorly utilized neurologist. The headache group visit model at the Cooper University Urban Health Institute was established to address some of these challenges.

Currently, group visit follow-up appointments are scheduled on a monthly basis. Some patients choose to skip months. If patients haven't come for a group visit in several months, staff make reminder phone calls. A patient may alternate between group visits and individual follow-up appointments if they wish. However, by alternating, they may lose the benefit of monthly access, since lag time for regular appointments is several months. As previously mentioned, all patients who are new to the neurology service are seen first on an individual basis outside the group by any neurologist in the practice. A thorough review of their medical record, a complete neurological examination and review of testing is conducted. At that point, a decision is made regarding appropriateness for the group visit setting. Those with complex medical histories (which might include other neurological diagnosis) or behavioral issues may be excluded from the group. This decision is left to the discretion of the referring neurologist.

Some patients attend headache group visits and also see a neurologist for other reasons, such as epilepsy management. If their non-headache neurological diagnosis is well controlled, (for example, if they have not had seizures in years), they may get their seizure medications renewed in the group visit. This would also require doing appropriate lab testing such as anti-epileptic drug blood level checks or periodic bone density screening during the group visit. If their epilepsy becomes uncontrolled, they will be referred to an epileptologist.

\section{Group visit location}

The space provided for group visits was custom constructed to accommodate a large conference room with U-shaped table where navigators and patients meet, one-on-one. Here, the physician will walk around the table to meet each patient once they have finished with the navigators. This room is linked to the adjoining educational room, where patients sit in chairs arranged in a circle formation; this design facilitates conversation and interaction. In this room, medical assistants will approach patients and obtain vital signs while they wait. Here, medical students and residents are encouraged to speak with patients and ask about their goals or address questions. After all patients have finished meeting with the neurologist, they return to the educational room for group discussion. Facilitators and/or guest speakers join the group arranged in a circle. A large projector screen is available here for meditation/yoga videos.

\section{Headache group visit outcomes}

Our group visit model has shown to improve lag time between follow-up visits, as it has allowed patients to come in monthly, as opposed to 4-month follow-up offered at individual appointments. This model also improved provider productivity as it has increased the number of patients the neurologist can see in a 2-hour span. In a regular clinic, patients are allotted 20-minute slots for follow-up; therefore, the provider is able to see 6 patients in a 2-hour span. During the 2-hour group visit, the provider is able to see up to 12 patients. On average, we currently see 7-12 patients. As eligible patients continue to be referred to the group, we expect to see an increase in the number of patients scheduled, and theoretically, an improvement in the financial feasibility.

The group has also provided patients with opportunity to interact with others with similar ailments, develop mutually supportive relationships, and compare experiences with various treatment modalities. There are no current data available from patient surveys or questionnaires. Initially, Migraine Disability Assessment (MIDAS) questionnaires were administered," but we discovered the scores to be less then helpful; many of the subtleties of the migraine experience were missed. The MIDAS assesses a patient's degree of disability attributable to migraine over a 3-month span. The questionnaire asks specifically objective questions such as "How many days of work or school did you miss?" and allows little room for patients to report psychological and social dysfunction. Many of the patients in our Camden population do not attend work or school. In the future, we plan to apply the Headache Impact Test (HIT-6) scoring system. ${ }^{12}$ HIT-6 assesses the subjective impact of headaches on a patient's quality of life over a 4-week span. The questionnaire asks questions pertaining to bodily pain as well as social, psychological, and cognitive dysfunction. The HIT-6 may be more appropriate for our patient population than the MIDAS, and may provide a clearer picture of patients' experiences. ${ }^{12}$ Anecdotally, patients have reported high satisfaction with the group visits. Additional outcomes such as headache pain reduction, financial gains, and drop-out rates are currently being evaluated.

\section{The evolving model of the group visit structure}

Our group visit was structured on the model developed by the diabetes group visit at Cooper University Hospital. ${ }^{13}$ Over the past few years, we have been growing in capacity and are now able to accommodate 7-15 patients per visit. Initially, providers in the navigator roles were clinical pharmacists and nurse practitioners. Over time, pharmacy students, medical students, licensed practical nurses, and physician assistants were added to drive down the cost. Training sessions have been designed for all staff to help prepare them to serve as navigators. During the sessions, common scenarios and all clinical interview questions are discussed. The pharmacy students are trained by the clinical pharmacist and the medical students are trained by the neurologist. Guest navigators, such as rotating neurology residents, receive brief orientation.

The group visit structure has been changing to improve the patient experience. Over the past few years, we have changed the order of events, for example we moved meditation/yoga from the first part of the session to the last part. We felt that the patients were more relaxed at the end and were able to enjoy the meditation and yoga more that way. Initially, the neurologist sat on one desk and the patients walked up to her after they finished with the navigator. Now, the neurologist walks around the room with a laptop, so that the navigator and patient teams stay in place. We have also evolved into an arrangement so that all patients and all navigators participate in the group discussion while sitting in chairs in a circle formation in the educational room. This creates a friendly environment where people 


\section{Special Report Migraine}

face each other, in addition to the neurologist. Additionally, we scheduled the injections for the last part of the visit. This encourages patients to stay for the entire group visit prior to receiving the injections.

Each modification was approved by a team made up of the neurologist, the navigators, and the Urban Health Institute director. The headache group visit model has proven to be an effective alternative to individual follow-up visits in our institute, as it expands therapeutic options for patients and offers a multi-faceted approach to the clinical encounter. Additionally, we have found that it increases physician productivity and enhances the cost-effectiveness of chronic illness management in this under-served patient population. We eagerly look forward to continued outcome improvement as patient satisfaction and self-ownership of their disease increases.
1. World Health Organization. Headache disorders. 2016. Available at: www.who.int/news-room/fact-sheets/detail/headachedisorders (accessed November 16, 2019)

2. Martelletti P, Birbeck GL, Katsarava Z, et al. The Global Burden of Disease Survey 2010. Lifting the burden and thinking outside-the-box on headache disorders. J Headache Pain. 2013;14:13

3. Headache Classification Committee of the International Headache society (IHS) The International Classification of Headache Disorders, 3rd edition. Cephalalgia. 2018;38:1-211.

4. Smith R. Impact of migraine on the family. Headache. 1998:38:423-6.

5. Mauser ED, Rosen NL. So many migraines, so few subspecialists: analysis of the geographic location of United Council for Neurologic Subspecialties (UCNS) certified headache subspecialists compared to United States headache demographics. Headache. 2014:54:1347-57.

6. Blumenfeld A, Tischio M. Center of excellence for headache care: group model at Kaiser Permanente. Headache. 2003:43:431-40

7. Winter AC, Berger K, Buring J, Kurth T. Associations of socioeconomic status with migraine and non-migraine headache. Cephalalgia. 2012;32:159-70.

8. Kryst S, Scherl E. A population-based survey of the social and personal impact of headache. Headache. 1994;34:344-50.

9. Buse DC, Manack A, Serrano D, et al. Sociodemographic and comorbidity profiles of chronic migraine and episodic migraine sufferers. I Neurol Neurosurg Psychiatry. 2010;81:428-32.

10. United States Census Bureau. Camden City,
New Jersey QuickFacts, 2015. Available at: www.census.gov/quickfacts/camdencitynewjersey (accessed November 16, 2019)

11. Stewart WF, Lipton RB, Dowson AJ, Sawyer J. Development and testing of the Migraine Disability Assessment (MIDAS) Questionnaire to assess headache-related disability. Neurology. 2001;56(Suppl. 1):S20-8.

12. Sauro KM, Rose MS, Becker WJ, et al. HIT-6 and MIDAS as measures of headache disability in a headache referral population. Headache. 2010;50:383-95.

13. Ganetsky VS, Brenner JC, Kaufman ST. Specialty group medical visits for patients with diabetes. Clin Diabetes. 2017:35:345-9. 